\title{
Resveratrol enhances prostate cancer cell response to ionizing radiation. Modulation of the AMPK, Akt and mTOR pathways
}

Ayesha Rashid ${ }^{1,2}$, Caiqiong Liu' ${ }^{1}$, Toran Sanli ${ }^{1,3}$, Evangelia Tsiani ${ }^{4}$, Gurmit Singh ${ }^{3}$, Robert G Bristow ${ }^{5}$, lan Dayes ${ }^{2}$, Himu Lukka ${ }^{2}$, James Wright ${ }^{2}$ and Theodoros Tsakiridis ${ }^{1,2^{*}}$

\begin{abstract}
Background: Prostate cancer (PrCa) displays resistance to radiotherapy (RT) and requires radiotherapy dose escalation which is associated with greater toxicity. This highlights a need to develop radiation sensitizers to improve the efficacy of RT in PrCa. lonizing radiation (IR) stimulates pathways of IR-resistance and survival mediated by the protein kinase Akt but it also activates the metabolic energy sensor and tumor suppressor AMP-Activated Protein Kinase (AMPK). Here, we examined the effects of the polyphenol resveratrol (RSV) on the IR-induced inhibition of cell survival, modulation of cell cycle and molecular responses in PrCa cells.

Methods: Androgen-insensitive (PC3), sensitive (22RV1) PrCa and PNT1A normal prostate epithelial cells were treated with RSV alone (2.5-10 $\mu \mathrm{M})$ or in combination with IR (2-8 Gy). Clonogenic assays, cell cycle analysis, microscopy and immunoblotting were performed to assess survival, cell cycle progression and molecular responses.
\end{abstract}

Results: RSV (2.5-5 $\mu \mathrm{M})$ inhibited clonogenic survival of PC3 and 22RV1 cells but not of normal prostate PNT1A cells. RSV specifically sensitized PrCa cells to IR, induced cell cycle arrest at G1-S phase and enhanced IR-induced nuclear aberrations and apoptosis. RSV enhanced IR-induced expression of DNA damage $(\gamma \mathrm{H} 2 \mathrm{Ax})$ and apoptosis (cleaved-caspase 3) markers as well as of the cell cycle regulators p53, p2 $1^{\mathrm{cip} 1}$ and p27 $7^{\mathrm{kip} 1}$. RSV enhanced IRactivation of ATM and AMPK but inhibited basal and IR-induced phosphorylation of Akt.

Conclusions: Our results suggest that RSV arrests cell cycle, promotes apoptosis and sensitizes PrCa cells to IR likely through a desirable dual action to activate the ATM-AMPK-p53-p2 $1^{\mathrm{cip} 1} / \mathrm{p} 27^{\mathrm{kip} 1}$ and inhibit the Akt signalling pathways.

Keywords: radio-sensitizers, clonogenic survival, cell cycle, ATM, p53, p21 cip1

\section{Introduction}

Radiotherapy is an effective therapy for localized prostate cancer ( $\mathrm{PrCa}$ ) but this disease is highly resistant to ionizing radiation (IR). Conventional radiotherapy doses up to 70 Gy show biochemical failure rates of $30 \%$ or more in localized disease [1], leading to a need for RT dose escalation, which is associated with rectal and bladder toxicity. Therefore, there is a need for rational development of effective radiosensitizers for PrCa.

\footnotetext{
* Correspondence: theos.tsakiridis@jcc.hhsc.ca

'Translational Radiation Biology Laboratory, Juravinski Cancer Centre, 699

Concession Street, Hamilton, Ontario, L84 5C2, Canada Full list of author information is available at the end of the article
}

The phosphatidylinositol 3-kinase (PI3k)-Protein kinase B/Akt (henceforth: Akt) pathway is known to promote proliferation, cell cycle progression and resistance to cytotoxic therapies in PrCa [2]. PI3k is an effector of the epidermal growth factor receptor (EGFR) [3], that leads to recruitment of Akt and its activators to plasma membrane. Akt is activated by phosphorylation on residues T308 and serine S473, both of which are required for activation [4]. T308 phosphorylation is mediated by the phosphoinoisitide-dependent kinase 1 (PDK1) [5] but the kinase mediating S473 phosphorylation (PDK2) is not clearly defined. Candidate kinases include the DNA damage sensor Ataxia Telangiectasia
Ciomed Central 
Mutated (ATM) [6]. Activated Akt mediates transcription of genes involved in survival and inhibition of those involved in apoptosis (see [2,7] for review). It promotes cell cycle progression through inhibition of the cell cycle regulators p53 [8] and the cyclin-dependent kinase inhibitors (CDKI) p $21^{\text {cip } 1}$ and p27 $7^{\text {kip } 1}[9,10]$. Furthermore, it regulates metabolic and nuclear processes through activation of the mammalian target-of-rapamycin (mTOR). Importantly, IR elicits cytoprotective responses mediated in part through activation of the PI3k-Akt pathway [11]. Akt is a mediator of radioresistance and PI3k-Akt pathway inhibitors are shown to enhance radiosensitivity of cancer cells $[7,12]$.

AMPK is a heterotrimeric enzyme that consists of an $\alpha$-catalytic and $\beta$-and $\gamma$-regulatory subunits [13]. It is a key regulator of carbohydrate and lipid metabolism and of proliferation in normal and cancer cells. AMPK detects an elevated AMP/ATP ratio in conditions of metabolic stress such as starvation and exercise [13] and promotes energy conservation by inhibiting protein synthesis, through mTOR inhibition while it also functions as a metabolic checkpoint to induce cell cycle arrest via p53 [14]. Recently, we showed that IR activates AMPK in human lung, breast and PrCa cells and suggested that AMPK participates in a signaling pathway involving ATM-AMPK-p53-p21 ${ }^{\text {cip } 1}$ leading to regulation of the cell cycle and survival [15].

RSV (3,4',5-trihydroxystilbene) is a polyphenolic phytoalexin with widely reported anti-aging and anti-cancer properties $[16,17]$. It inhibits cancer cell proliferation and is suggested to enhance radiation responses $[18,19]$. RSV has also been reported to increase metabolic rate and reduce fat mass in wild-type mice but not in AMPK $\alpha$ subunit knockout mice [20]. Further, it was shown to suppress tumor growth and metastasis in the mouse Lewis lung carcinoma model [21]. RSV is known to regulate both Akt and AMPK [22,23] but the effects of this compound on the two signaling pathways have not been studied in radiated cells.

Here, we investigated the polyphenol RSV due to the reported ability of this natural compound to modulate both the radioresistance-mediating Akt and the tumour suppressor AMPK pathways [24,25].

\section{Materials and methods}

Cell Lines and Cell Culture

Human PrCa (PC3, 22RV1) and normal prostate epithelial (PNT1A) cell lines were obtained from American Tissue Culture Collection (Manassas, VA, U.S.A.). Cells were maintained at $37^{\circ} \mathrm{C}$ in RPMI media supplemented with 10\% (vol/vol) Fetal Bovine Serum (FBS) and 1\% (vol/vol) antibiotic-antimycotic (Invitrogen, Burlington, ON, Canada).

\section{Reagents and Antibodies}

Rabbit polyclonal antibodies against total Akt (T-Akt), phosphorylated-(P)-(S473)-Akt, P-(T308)-Akt, P-mTOR, total AMPK (T-AMPK), P-(Thr172)-AMPK, T-ATM, P(S1981)-ATM, P-(S139)- $\gamma$ H2Ax, mouse monoclonal antibodies against p53, p21 ${ }^{\mathrm{cip} 1}, \mathrm{p} 27^{\mathrm{kip} 1}$, actin, an anti- $\alpha$ tubulin antibody conjugated to Alexa Fluor 488 as well as horseradish peroxidase (HRP)-conjugated IgG secondary anti-rabbit and anti-mouse antibodies were from New England Biolabs (Mississauga, ON, Canada). Hoechst 33258 was from Sigma (Toronto, ON, Canada). RSV and the KuDos Pharma ATM inhibitor KU55933 were from Calbiochem (Mississauga, ON, Canada). Anti- $\alpha 1$ and $-\alpha 2$ AMPK siRNA transfection kit was obtained from Qiagen (Mississauga, Ontario, Canada).

\section{Treatments}

Cells were treated with $2-8$ Gy IR using a ${ }^{60}$ Co clinical unit. For combined RSV or KU55933 and IR treatments, cells were kept at $37^{\circ} \mathrm{C}$ with the indicated agent for $1 \mathrm{~h}$ prior to IR treatment. Cells were incubated for $1 \mathrm{~h}$ following IR exposure, unless otherwise indicated. For cell cycle and clonogenic assays cells were exposed to the treatment agents throughout the experiments (as indicated in figure legends).

\section{siRNA AMPK $\alpha$ Subunit Knockdown}

Cells were incubated with a mixture of human siRNA sequences against the $\alpha 1$ and $\alpha 2$ AMPK subunits using HiPerFect vehicle for 72 hours as per the manufacturer's protocol.

\section{Clonogenic Assays}

Clonogenic assays were performed as described earlier [15]. Cells (500-1000) were seeded in triplicates and allowed to adhere overnight, then were incubated with RSV followed by IR treatment (0-2 Gy) followed by incubation for 7-10 days. Cells were then fixed and stained with $0.05 \%$ methylene blue and colonies (> 50 cells) were counted. Results are expressed as the surviving fraction compared to untreated control.

\section{Immunoblotting (IB)}

IB was performed as described earlier [26]. Approximately $5 \times 10^{5}$ cells were seeded in 6-well plates. After treatment, cells were washed, lysed and twenty micrograms of protein were subjected to IB.

\section{Immunofluorescence Microscopy}

Cells $\left(2 \times 10^{5}\right)$ were seeded onto glass coverslips and were incubated without or with RSV $(5 \mu \mathrm{M}) 1 \mathrm{~h}$ prior to IR treatment (8 Gy). Ninety six hours after IR exposure, cells were washed lightly with PBS and fixed with 
3\% paraformaldehyde/PBS/0.2\% Triton X-100 for 20 minutes. Cells were stained with Hoechst 33258 (20 $\mu \mathrm{M})$ nuclear stain or anti- $\alpha$-tubulin antibody conjugated to Alexa Fluor 488 (1:100 dilution) (followed by Hoechst 33258 nuclear staining) and examined by fluorescence microscopy. Four hundred cells were evaluated for nuclear aberrations (fragmentation, micro-nuclei and multi-nucleation) in four representative areas of each slide in 3 independent experiments.

\section{Cell Cycle Analysis}

$\left(1 \times 10^{6}\right.$ cells $\left./ \mathrm{mL}\right)$ were seeded in 10 -cm dishes. After treatment, cells were trypsinized, washed, fixed with $70 \%$ ethanol and stored overnight at $-20^{\circ} \mathrm{C}$ followed by washing and staining with a solution containing $100 \mu \mathrm{L}$ Triton X-100 and $50 \mu \mathrm{g} / \mathrm{mL}$ propidium iodide. Cells were subjected to flow cytometric cell cycle analysis using a Beckman Coulter Epics XL flow cytometer.

\section{Statistical Analysis}

Data are expressed as the mean \pm standard error (SE). Statistical analysis was performed using unpaired t-test with SPSS v16.0 software (SPSS, Chicago, IL). Statistical significance was considered at $\mathrm{p}<0.05$.

\section{Results}

\section{RSV inhibits PrCa cell survival}

As a single agent, RSV effectively inhibited survival of both PC3 and 22RV1 PrCa cells with significant inhibition achieved at even the lower doses of 2.5 and $5 \mu \mathrm{M}$ $(\mathrm{p}<0.05)$ (Figure $1 \mathrm{~A})$. The $\mathrm{IC}_{50}$ values were approximately $10 \mu \mathrm{M}$ and $2.5 \mu \mathrm{M}$ for PC3 and 22RV1 cells, respectively. The PC3 response to RSV was dose-dependent. RSV $2.5 \mu \mathrm{M}$ significantly decreased survival in $22 \mathrm{RV}-1$ cells to $40 \pm 3.06 \%$ of control without any further decrease at higher doses. In contrast, PNT1A normal epithelial cells were less responsive to RSV showing only 5 and $10 \%$ inhibition of survival at 2.5 and $5 \mu \mathrm{M}$, respectively (Figure 1A). Only the higher dose of $10 \mu \mathrm{M}$ caused significant decrease in normal epithelial PNT1A cell survival (to $60 \pm 12.26 \%$ of control) ( $\mathrm{p}<$ $0.05)$. PTN1A cells were used here as a non-malignant control.

\section{IR-induced inhibition of PrCa cell survival}

PC3 cells showed greater resistance to IR alone compared to 22RV1 cells (surviving fractions after 2 Gy (SF2) of $60 \pm 5.30 \%$ vs $40 \pm 3.53 \%$, respectively, Figure 1B). Their IR sensitivity was similar to that of PNT1A cells (SF2 of $62.9 \pm 2.26 \%$, Figure 1B). In clonogenic assays we opted to use the standard therapeutic IR dose of $2 \mathrm{~Gy}$ and not high doses of IR, for clinical relevance and because higher doses (i.e. 6-8 Gy) were highly toxic to PrCa cells in agreement with previous reports [27].
RSV enhances the IR-induced inhibition of clonogenic survival in PrCa cells

RSV augmented further the IR-induced inhibition of survival in both PC3 and 22RV1 PrCa cells. RSV (2.5 and $5 \mu \mathrm{M})$ decreased survival of IR-treated PC3 cells by $(13.8 \pm 0.09 \%$ and $20.4 \pm 0.9 \%$, Figure 1B). RSV $5 \mu \mathrm{M}$ caused only a minor reduction of SF2 in 22RV1 cells (non-significant) but at $10 \mu \mathrm{M}$ the drug induced a significant 2.5-fold inhibition in SF2. RSV $(5 \mu \mathrm{M})$ pre-treatment did not decrease further SF2 of PNT1A prostate epithelial cells but this was achieved when RSV was increased to $10 \mu \mathrm{M}$ (Figure $1 \mathrm{~B}$ ).

To better demonstrate the potential clinical benefit of RSV in combination with radiotherapy, we used SF2 values to estimate tumour cell survival after 40 fractions of radiotherapy ( 2 Gy each), which consists a standard approach in clinical PrCa management [1]. We determined (SF2) ${ }^{\mathrm{n}}$ as the tumour cell fraction surviving after " $n$ " fractions of radiotherapy. Assuming iso-effectiveness of all fractions, we plotted the projected tumour survival over 40 consecutive fractions (0-80 Gy) (Figure 1C). As typical human prostates range between $35-100$ gr $\left(5-10 \times 10^{10}\right.$ cells $)$ we plotted projected tumour size reductions (tumour killing) on a scale of $1-1 \times 10^{-11}$. Figure $1 \mathrm{C}$ estimates that RSV $(5 \mu \mathrm{M})$ may be able to reduce the dose of radiotherapy needed to eliminate 22RV1 type (hormone-sensitive) PrCa tumours (from about 47 to 40 Gy) and may be able to eliminate PC3 type (hormoneinsensitive) PrCa tumours (with about 54 Gy) that would otherwise be incurable with doses even higher than $80 \mathrm{~Gy}$. Further, this approach also demonstrated the complete lack of radio-sensitization of normal epithelial cells (PNT1A) by $5 \mu \mathrm{M}$ RSV. Based on these results, we pursued all subsequent studies with $\mathrm{PrCa}$ cells alone and with doses of $2.5-5 \mu \mathrm{M}$ RSV.

RSV inhibits IR-mediated cell cycle arrest and induces cell accumulation at G1-S and sub-G1 phases consistent with apoptosis

We utilized the 22RV1 cell line in our cell cycle analysis experiments because it is a cloned cell line [28] consisting of a more isogenic population of cells than PC3 cells, and is hence appropriate for cell cycle analysis. As expected, IR induced an arrest of cells at the G2-M interphase (Control: 13.4\% vs. IR: 31.7\%, Figure 2) but pre-treatment with RSV $(5 \mu \mathrm{M})$ prevented this event (RSV+IR: $8.3 \%$ vs. $31.7 \%$ for IR, Figure 2). RSV caused accumulation of radiated cells into the G1-S phases of the cycle (IR: $58 \%$ vs. RSV + IR: 70.7\%) and increased the population of cells in the sub-G1 region by 2 -fold compared to those treated with IR alone (IR: $10.3 \%$ vs. RSV + IR: $21.0 \%$ ), indicating induction of apoptosis. 


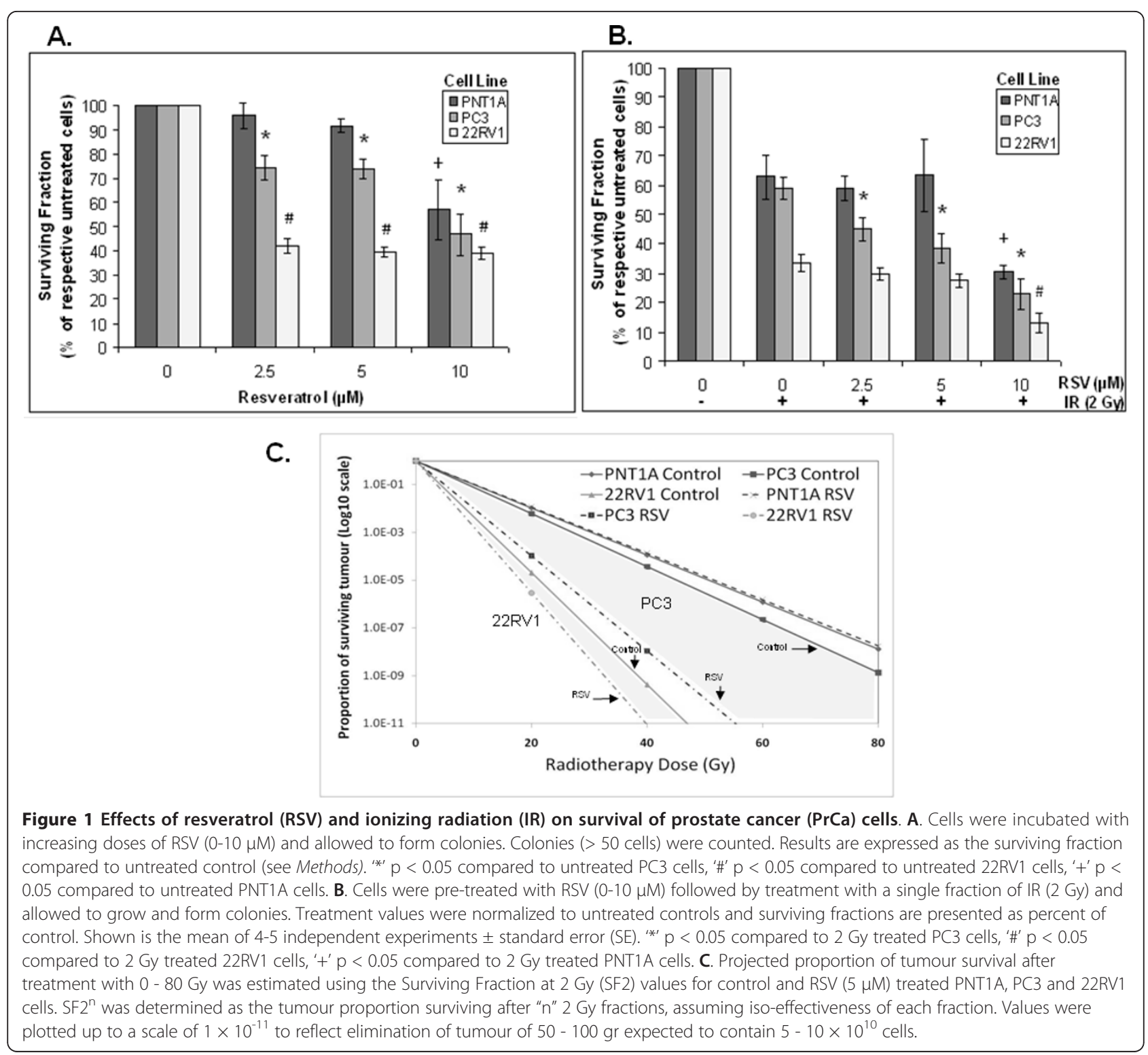

RSV enhances the IR induction of cell cycle inhibitors

To examine whether the effects of RSV on IR regulation of the cell cycle were associated with enhancement of molecular pathways of cell cycle regulation, we examined the expression of p53 and the cyclin-dependent kinase inhibitors (CDKI) $\mathrm{p} 21^{\mathrm{cip} 1}$ and $\mathrm{p} 27^{\mathrm{kip} 1}$. Figure $2 \mathrm{~B}$ shows that IR induced expression of both p53 and the CDKIs in 22RV1 cells and that RSV enhanced this further. Similar results were obtained for the two CDKIs in p53-null PC3 cells.

\section{RSV induces expression of apoptotic markers and nuclear aberrations}

Using immunoblotting with a cleaved caspase-3-specific antibody, we examined the effects of RSV and IR on the levels of this established apoptosis marker. IR increased caspase-3 cleavage within 1 hour of IR exposure in both PC3 and 22RV1 cells (Figure 3A). Pre-treatment with RSV $(2.5$ and $5 \mu \mathrm{M})$ enhanced the IR-induced cleaved caspase-3 levels in a dose-dependent fashion.

To evaluate morphological markers of apoptosis, cells were stained with Hoechst 33258 and examined with microscopy. Cells with nuclear aberrations such as fragmentation, micro-nuclei and multi-nucleation (polysomy), which are markers predictive of apoptosis and/or mitotic cell death (mitotic catastrophe), were counted in each treatment condition. Figure 3B shows representative images of treated PC3 cells that were either stained with Hoechst 33258 alone (low magnification, Figure 3B) or additional immunostaining of tubulin (high 


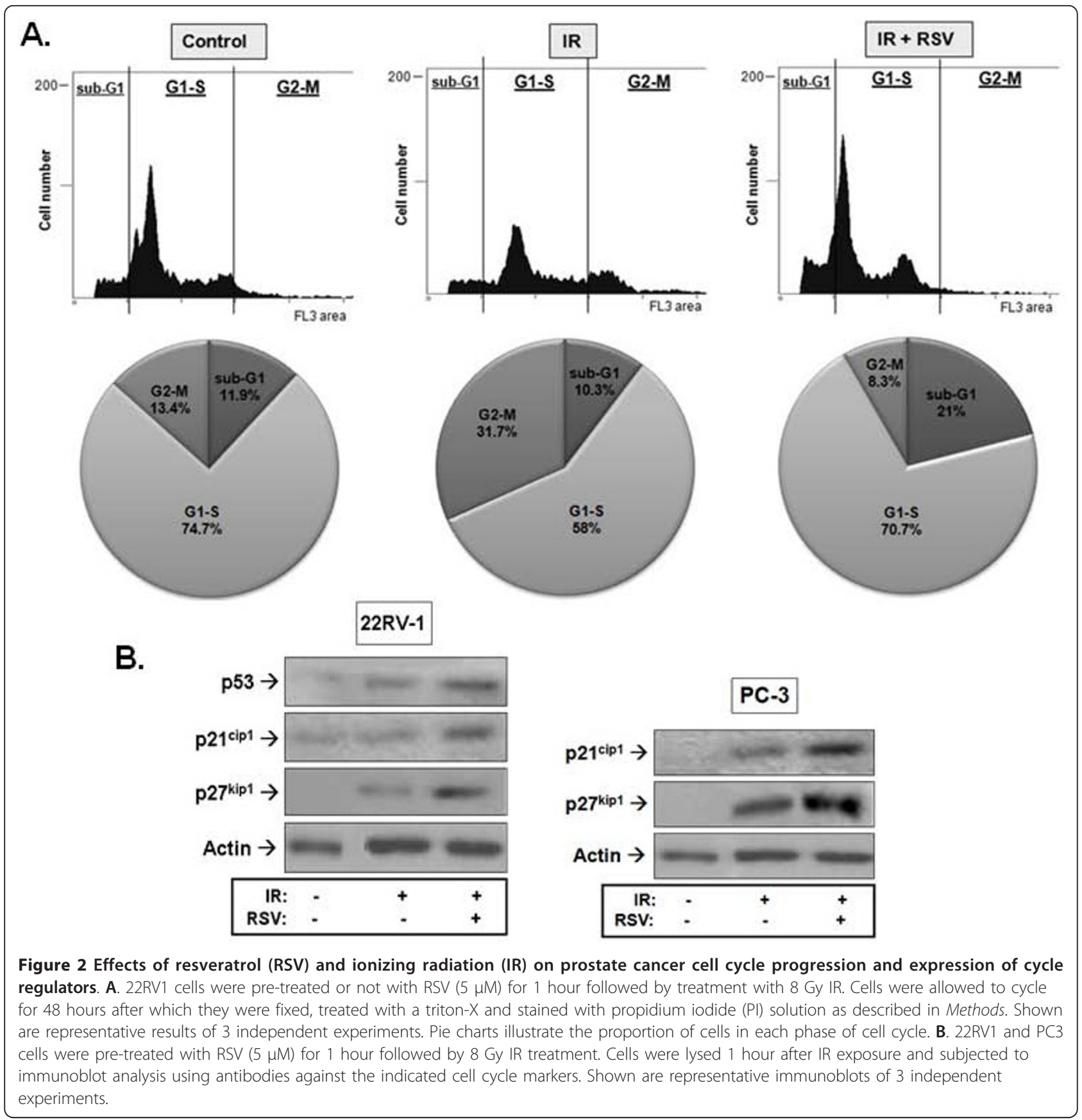

magnification), which was utilized to better demonstrate the effects of the treatments on nuclear and cellular morphology (Figure 3C). The results of four independent experiments (as in Figure 3B) were quantitated (as described in Methods) and are shown in Figure 3D). IR alone induced significant nuclear aberrations $(43 \pm$ $2.18 \%$, Figure $3 \mathrm{~B})$, while RSV alone $(5 \mu \mathrm{M})$ increased nuclear abnormalities by $6 \%$ (Figure 3B). However, RSV significantly enhanced the IR-induced nuclear aberrations to $70 \pm 3.11 \%$ (an increase of 27\%, Figure 3D) of total cell counts and reduced overall cellular viability (Figure 3B).

Effects of RSV on molecular pathways of radio-resistance and tumour suppression

RSV inhibits basal and IR-induced levels of phosphorylated Akt

We examined the effects of RSV on the well-described radio-resistance pathway of Akt-mTOR. We observed that IR alone induced a significant time-dependent 


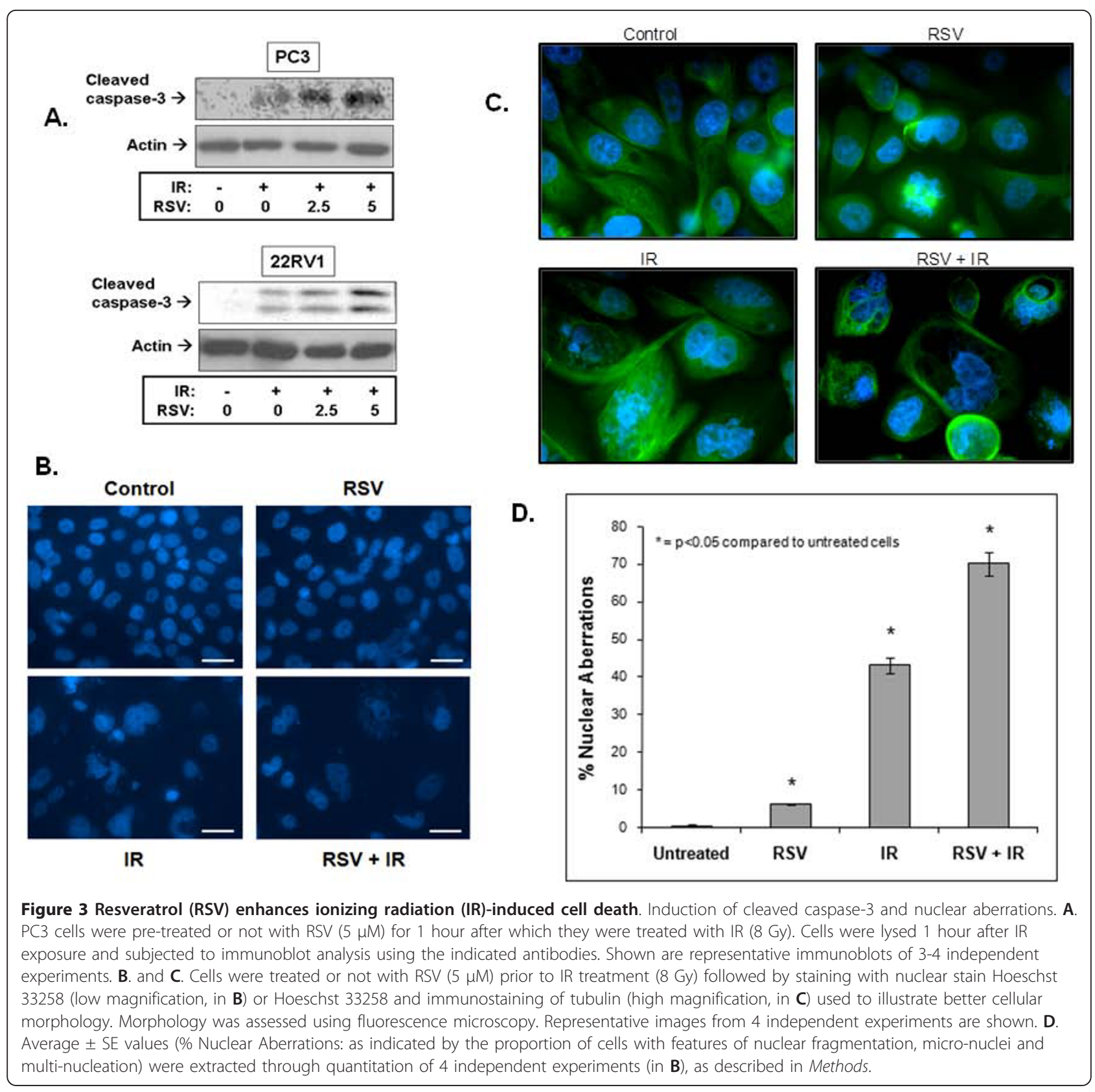

increase in the levels of phosphorylated Akt (P-Akt) (both S473 and T308 sites) in PC3 cells, with no effects on total levels of the protein (Figure 4A). Highest levels of P-Akt were reached 30 minutes to 1 hour after IR exposure. Akt phosphorylation was associated with increased activity of this enzyme indicated by the IRinduced phosphorylation of mTOR, a key effector of Akt (Figure 4A). Similar results were obtained in 22RV1 PrCa cells (results not shown).

Treatment of PC3 and 22RV1 cells with RSV inhibited basal levels of P-Akt (Figure 4B). Of note, the images shown were obtained after significantly longer film exposure to allow demonstration of the effects of RSV on basal Akt phosphorylation which are significantly lower than in radiated cells (as shown in Figure 4A and 4C). Pre-treatment with RSV led to significant inhibition of IR-induced P-Akt with no effects on total levels of the protein (Figure 4C). Although IR exposure led to an increase in P-Akt, RSV pre-treatment $(2.5$ and $5 \mu \mathrm{M})$ significantly reduced and/or prevented IR-induced phosphorylation of Akt. The effects of RSV on IR-induced Akt phosphorylation on S473 were quantitated in four independent experiments (Figure 4D). Similar results were obtained for Akt T308 phosphorylation. A 
A.

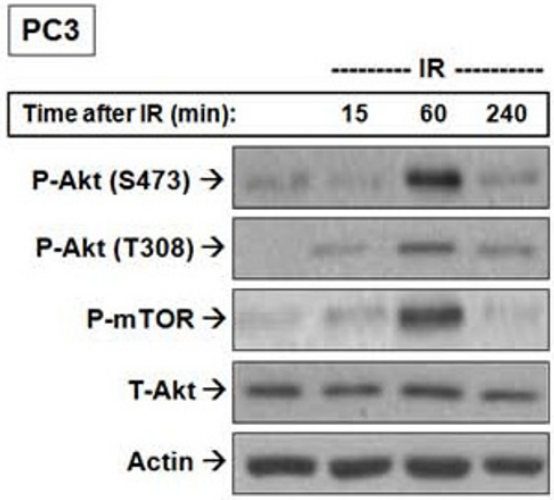

C.
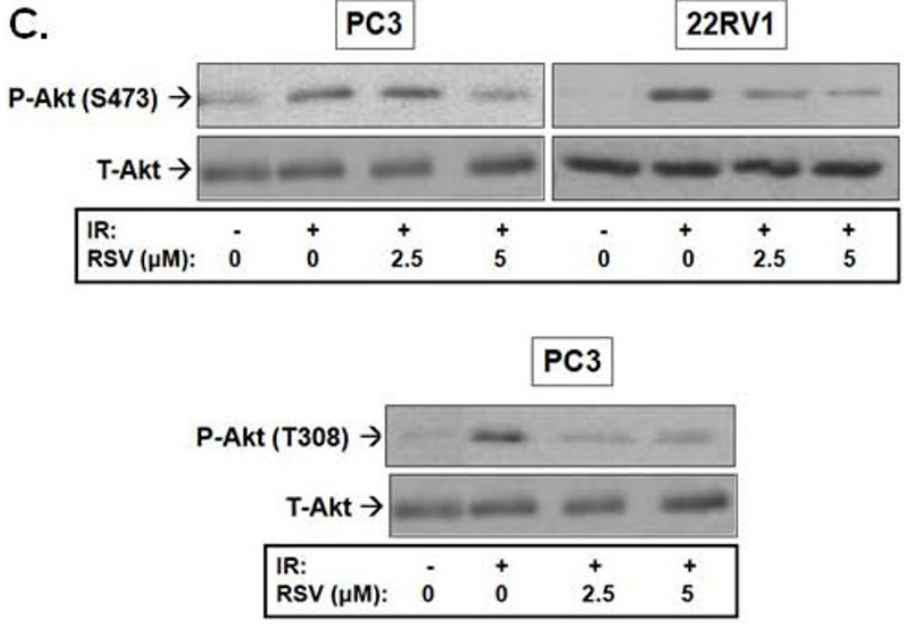

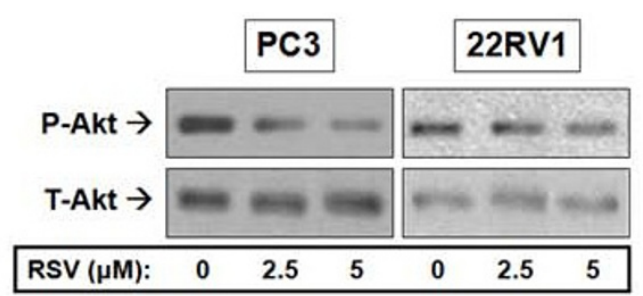

D.
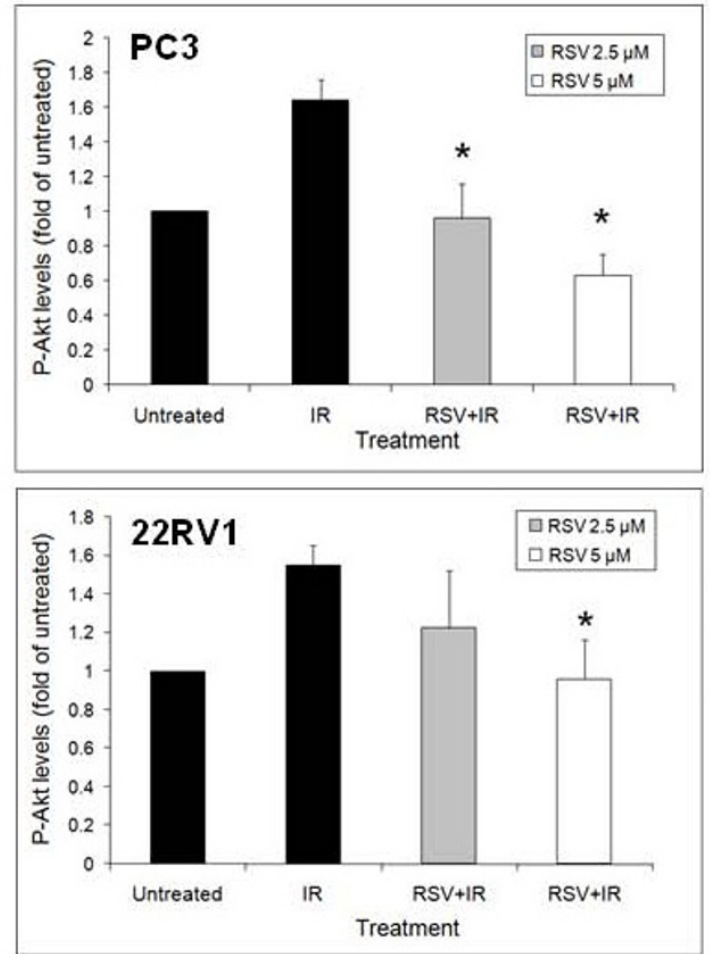

Figure 4 Resveratrol (RSV) inhibits basal and ionizing radiation (IR)-induced Akt phosphorylation. A. Effects of IR alone: PC3 cells were treated with 0 or $8 \mathrm{~Gy} I R$ and lysed according to the indicated time points following IR exposure. Then cells were subjected to immunoblot analysis. B. RSV inhibits basal Akt phosphorylation: PC3 and 22RV-1 cells were treated with RSV (0,2.5 or $5 \mu \mathrm{M})$ for 1 hour and subjected to lysis and immunoblot analysis using the indicated antibodies. C. RSV inhibits IR-induced Akt phosphorylation. PC3 and 22RV-1 cells were pre-treated with the indicated concentrations of RSV 1 hour prior to IR treatment. Cells were lysed 1 hour after IR exposure and subjected to immunoblot analysis. Shown are representative immunoblots of 4 independent experiments. D. Densitometric quantitation of Akt (S473) phosphorylation and total Akt was performed. P-Akt was normalized to T-Akt levels and presented as fold change compared to untreated control cells (mean \pm SE) from 3-4 independent immunoblotting experiments as in $\mathbf{C}) .{ }^{*} p<0.05$ compared to cells treated with IR alone.

representative immunoblot from PC3 cells is shown (Figure 4C).

\section{RSV and IR effects on AMPK}

We reported earlier that IR activates AMPK in lung, breast and prostate cancer cells [15]. Similarly, we observed here that IR induced a robust phosphorylation of AMPK on Thr172 of the catalytic $\alpha$ subunit that was detectable within 15 min. Highest levels of phosphorylated AMPK (P-AMPK) were detected 1 hour after IR exposure and returned to almost basal levels 4 hours after radiation (Figure 5A). This was associated with activation of AMPK indicated by the detected phosphorylation of the AMPK substrate Acetyl CoA Carboxylase
(ACC). RSV induced a robust activation of AMPK in both PC3 and 22RV1 cells at both 2.5 and $5 \mu \mathrm{M}$ (Figure $5 B)$. Furthermore, RSV pre-treatment enhanced significantly the IR-induced AMPK phosphorylation in both 22RV1 and PC3 cells (Figure 5C). Total AMPK levels remained unchanged after both IR and RSV treatments. The results of 3-4 independent experiments were quantitated and are shown in Figure 5D.

\section{The pathway of radiation activation of Akt and AMPK Akt regulation}

Recent reports suggest that Akt activation by IR may be regulated by ATM [29]. We examined this notion in 
A.
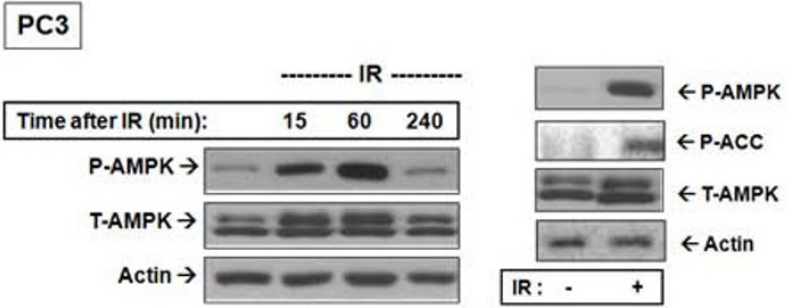

B.

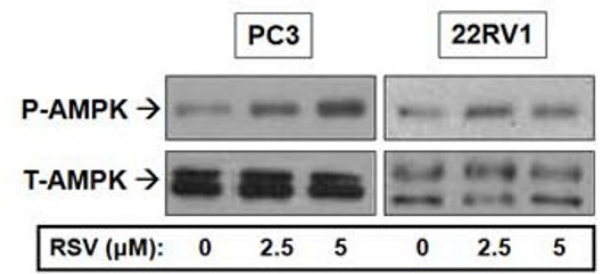

c.

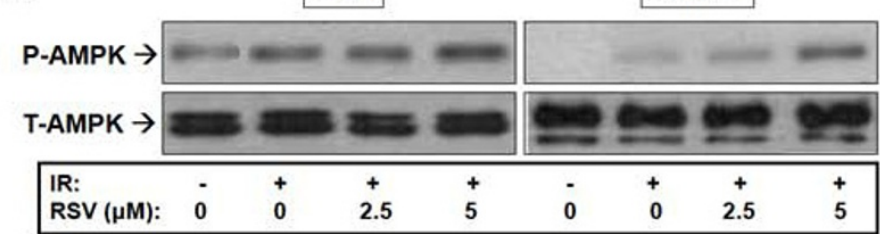

D.
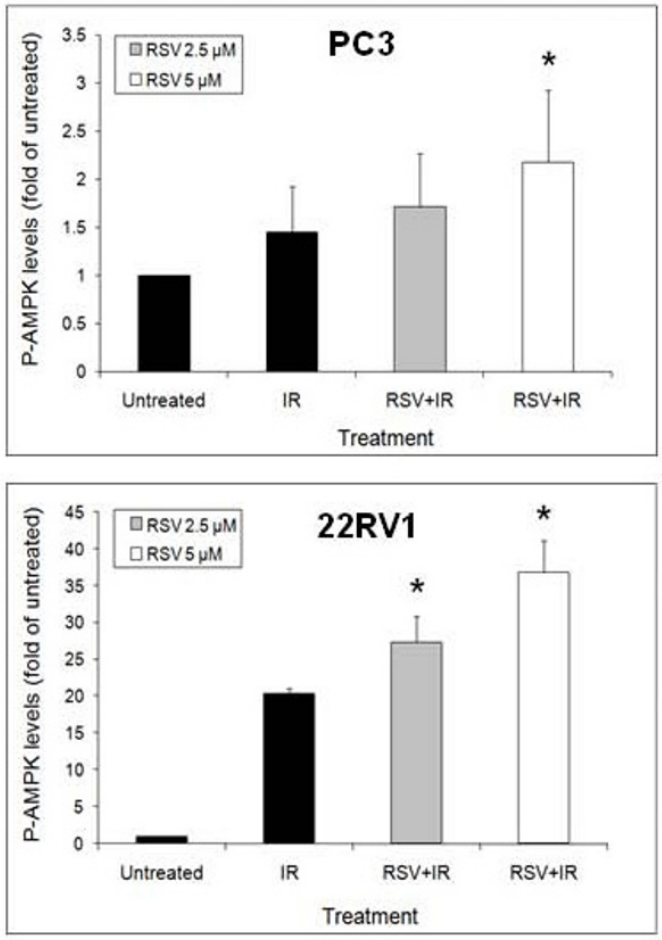

Figure 5 Resveratrol (RSV) enhances basal and ionizing radiation (IR)-induced AMPK phosphorylation. A. Effects of IR alone: PC3 cells were treated with 0 or 8 Gy IR and lysed at the indicated time points after IR exposure. The adjacent panel shows PC3 cells treated with 8 Gy IR, which were lysed 1 hour after IR treatment. Samples were subjected to immunoblot analysis using the indicated antibodies. B. RSV enhances basal AMPK phosphorylation: PC3 and 22RV-1 cells were treated with the indicated concentrations of RSV for 1 hour followed by lysis and immunoblotting analysis. C. RSV enhances IR-induced AMPK phosphorylation. PC3 and 22RV-1 cells were pre-treated with the indicated concentrations of RSV 1 hour prior to 8 Gy IR treatment. Cells were lysed 1 hour after IR exposure and subjected to immunoblotting using indicated antibodies. Shown are representative immunoblots of at least 3-4 independent experiments. D. Densitometry-based quantitation. Density values of P-AMPK were normalized to T-AMPK levels and presented as fold change compared to untreated control cells (mean \pm SE) from 2-3 independent experiments in $\mathbf{C}$. is shown. ${ }^{*} p<0.05$ compared to cells treated with $\mathbb{R}$ alone.

PrCa cells using the ATM-specific inhibitor KU55933 [30]. Pre-incubation with KU55933 prevented IRinduced ATM phosphorylation but also inhibited IR phosphorylation of Akt at S473 and activation of its kinase activity as indicated by reduced phosphorylation of mTOR (Figure 6A).

\section{AMPK Regulation}

We attempted to verify, in PrCa cells, our earlier observations in lung cancer cells [15] of AMPK participation in an ATM-AMPK-p53/p21 ${ }^{\text {cip } 1}$ pathway activated by IR. We observed a robust phosphorylation of ATM and AMPK as well as induction of $\mathrm{p} 21^{\mathrm{cip} 1}$ in PC3 PrCa cells in response to IR. IR induced ATM and AMPK phosphorylation and $\mathrm{p} 21^{\mathrm{cip} 1}$ induction were all inhibited by treatment with KU55933 (Figure 6B). To verify that AMPK truly acts as a mediator of $\mathrm{p} 53 / \mathrm{p} 21^{\text {cip } 1}$ induction in response to IR in PrCa cells, we used wild-type p53 expressing 22RV1 cells to perform AMPK knockdown experiments. siRNA knockdown of both $\alpha 1$ and $\alpha 2$ subunits of AMPK blocked p53 and p21 cip1 induction by IR (Figure 6C). Interestingly, RSV pre-treatment enhanced IR-induced phosphorylation of ATM and of its substrate histone $\mathrm{H} 2 \mathrm{Ax}(\gamma \mathrm{H} 2 \mathrm{Ax})$, as well as phosphorylation of AMPK and induction of $\mathrm{p} 21^{\mathrm{cip} 1}$.

\section{Discussion}

\section{IR and RSV effects on PrCa cell clonogenic survival}

RSV inhibits survival and proliferation of cancer cells as a single agent and induces radiosensitization in human cervical cancer cells [19]. Similarly, we observed that at low doses (2.5-10 $\mu \mathrm{M})$ RSV inhibited clonogenic survival of PrCa cells. Several studies have reported $\mathrm{IC}_{50}$ values for cell growth inhibition by RSV in the range of 5 to $10 \mu \mathrm{M}[31,32]$. Free RSV has a low bioavailability invivo as it is rapidly metabolized to glucoronide and sulfate conjugates [32]. A human study reported plasma concentrations of free RSV of $21 \mathrm{nM}$ after oral dose of $25 \mathrm{mg}$ RSV [32]. However, all combined RSV 


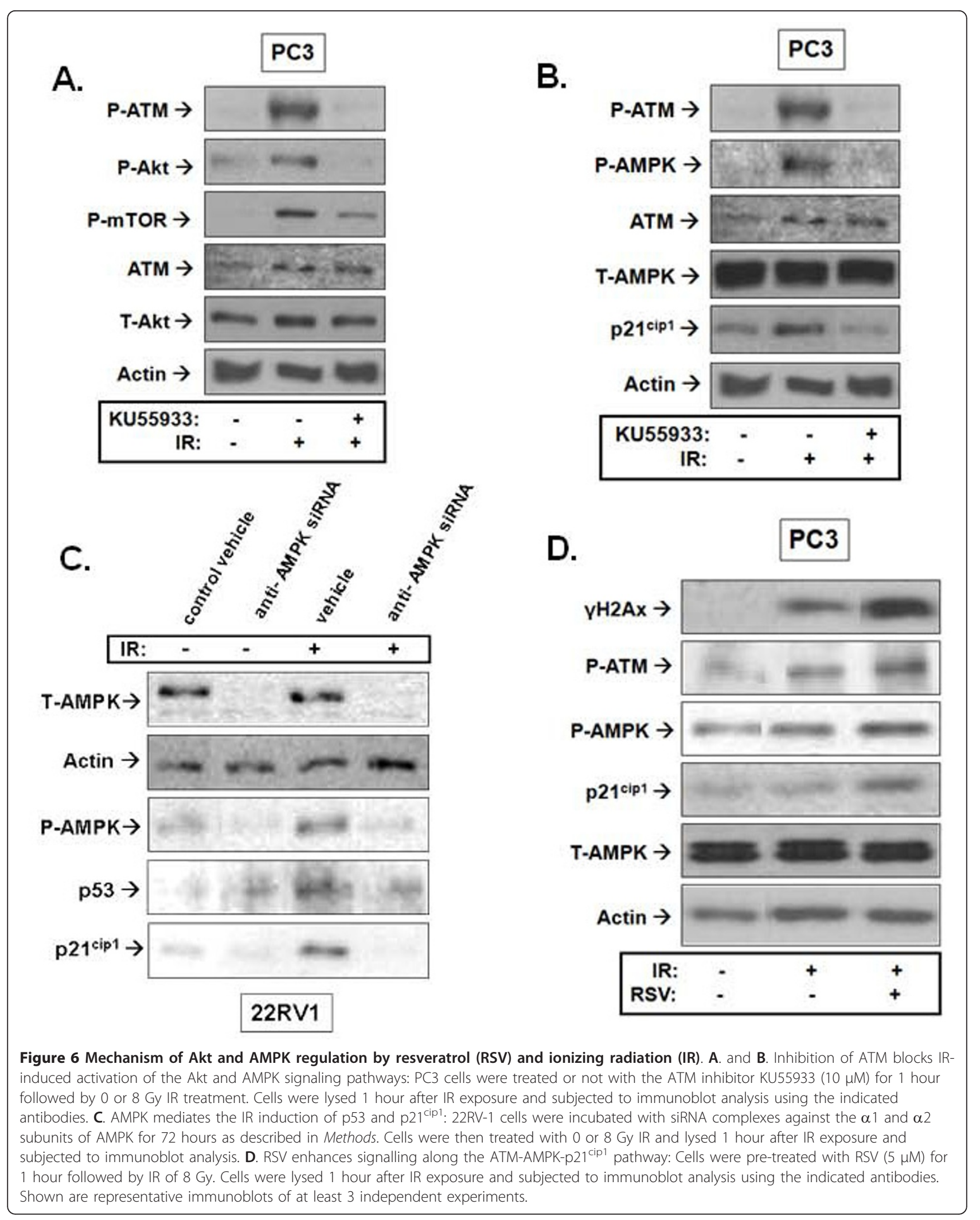


metabolites were reported to reach about $2 \mu \mathrm{M}$ [16]. For this, we pursued our studies with low RSV concentrations $(2.5-10 \mu \mathrm{M})$.

As a single agent, RSV inhibited more potently survival of 22RV1 compared to PC3 cells but the response of 22RV1 cells did not display dose-dependence. Overall, PC3 cells displayed greater resistance to both IR and RSV alone, consistent with other studies [33]. PC3 cells are deficient in key tumour suppressors including PTEN and p53 [34]. Lack of PTEN allows aberrant Akt activation [35], which in combination with the lack of p53, may confer such cells a survival advantage and IR- and RSV-resistance. PNT1A cells were less responsive to RSV, indicating a potential for this drug to achieve a positive therapeutic ratio in-vivo. RSV inhibited significantly PNT1A cells at $10 \mu \mathrm{M}$ and for that we focused our work on 2.5 and $5 \mu \mathrm{M}$ RSV.

We demonstrated that RSV can sensitize PrCa cells to IR (Figure 1). Concentrations of RSV (2.5 and $5 \mu \mathrm{M})$, similar to those that can be achieved in human serum, enhanced the cytotoxicity of a conventional RT fraction (2 Gy) in PrCa cells without additional toxicity to normal epithelial cells. The potential clinical utility of our finding is illustrated in Figure 1C, which suggests that low RSV doses have the potential to reduce the dose of radiotherapy required to treat human hormone- and radiation-sensitive $\mathrm{PrCa}$ and may be able to make curable hormone- and radiation-insensitive tumours that may otherwise be incurable with even modern dose escalated radiotherapy. This notion needs to be verified in in-vivo models of human PrCa.

\section{Regulation of cell cycle and apoptosis}

RSV is reported to arrest PrCa and other cells at G0-G1 and/or S-phases of the cycle leading to senescence $[36,37]$ and cause p2 $1^{\text {cip } 1}$-mediated G1-phase arrest and apoptosis in A431 cells [38]. Consistently, we observed a significant arrest of radiated PrCa cells at G1-S (Figure 4). RSV's effects on survival were additive to those of IR but the two agents mediate different regulation of cell cycle. Whereas IR induces G1-S and more so G2-M cycle arrest, RSV prevented the latter (Figure 2), likely due to induction of an earlier checkpoint (G1-S). The potentiation of IR-induced expression of p53 and CDK inhibitors $\mathrm{p} 21^{\mathrm{cip} 1}$ and $\mathrm{p} 27^{\mathrm{kip} 1}$ by RSV (Figure $2 \mathrm{~B}$ ), known to regulate the G1 and S phase checkpoints, may provide a molecular pathway of action for RSV's induction of the G1-S phase arrest seen in our studies.

RSV also caused re-distribution of cells into the subG1 or apoptotic range that was associated with cleavage of caspase- 3 and induction of extensive nuclear aberrations (Figure 3). Apoptosis appears to be the primary mode of cell death induced by RSV. Studies demonstrated such cell death effects in PrCa cells through activation of caspase-, p53-, or Fas ligand-dependent pathways $[39,40]$. In our studies, IR-mediated nuclear damage showed early signs of mitotic catastrophe and RSV potentiated such nuclear aberrations (Figure 3C-D). Mitotic cell death predominates in cells with defects in cycle checkpoints that prevent cycle arrest and DNA repair when cells are exposed to genotoxic stress such as IR [41]. The features of mitotic catastrophe seen in our studies in PC3 cells (p53-null) after RSV and IR treatments are consistent with those observed earlier in other p53-null cancer models [41].

\section{Modulation of the Akt and AMPK pathways}

IR and RSV exert opposite effects on Akt. IR mediated Akt phosphorylation on T308 and S473 and enzymatic activation indicated by mTOR phosphorylation (Figure 4A). This suggests activation of both PDK1 and PDK2 activity by IR. However, we observed that RSV is an effective inhibitor of this radioresistance-associated pathway (Figure 4). Inhibition of IR-induced T308 and S473 phosphorylation by RSV suggests an ability to regulate both PDK1 and PDK2. The enhancement of IR-induced cell death by RSV may indeed be mediated through modulation of Akt and its downstream targets as suggested earlier for uterine cancer [25] and PrCa cells [42].

Using the ATM-specific inhibitor KU55933, [30] we observed that ATM mediates IR-induced Akt S473 phosphorylation and activation, suggesting that ATM may indeed be the IR responsive PDK2, as suggested earlier [6]. However, despite inhibition of Akt, RSV enhanced ATM phosphorylation and activation indicated by histone $\mathrm{H} 2 \mathrm{Ax}$ phosphorylation $(\gamma \mathrm{H} 2 \mathrm{Ax})$ (Figure $6 \mathrm{D})$. This points to a complex regulatory action of RSV to enhance ATM activation but inhibit its PDK2 action on Akt downstream. This concept needs to be explored further in future studies.

We aimed to evaluate, in PrCa cells, the DNA damage responsive ATM-AMPK-p21 ${ }^{\text {cip } 1}$ pathway we proposed earlier in lung cancer cells [15] and found that IR indeed mediates AMPK activation downstream of ATM (Figure 6B). We observed that AMPK mediates p53 and p2 $1^{\text {cip } 1}$ induction in response to IR in PrCa cells (Figure 6C). RSV and IR stimulated phosphorylation/activation of AMPK, in both PC3 and 22RV1 PrCa cells, which was associated with induction of $\mathrm{p} 21^{\mathrm{cip} 1}$ and $\mathrm{p} 27^{\mathrm{kip} 1}$ (Figure 6D). Although, we did not examine whether the latter is regulated by AMPK activity, this notion is well described by other studies [43]. The enhancement of IRstimulated ATM activity by RSV provides a framework for upregulation of the ATM-AMPK-p2 ${ }^{\text {cip } 1}$ pathway in RSV-treated cancer cells and a rationale for the observed inhibition of cell cycle and survival. This notion is supported by observations that the AMPK inhibitor compound $C$ inhibits IR-induced cytotoxicity in $\mathrm{PrCa}$ (results not shown). 


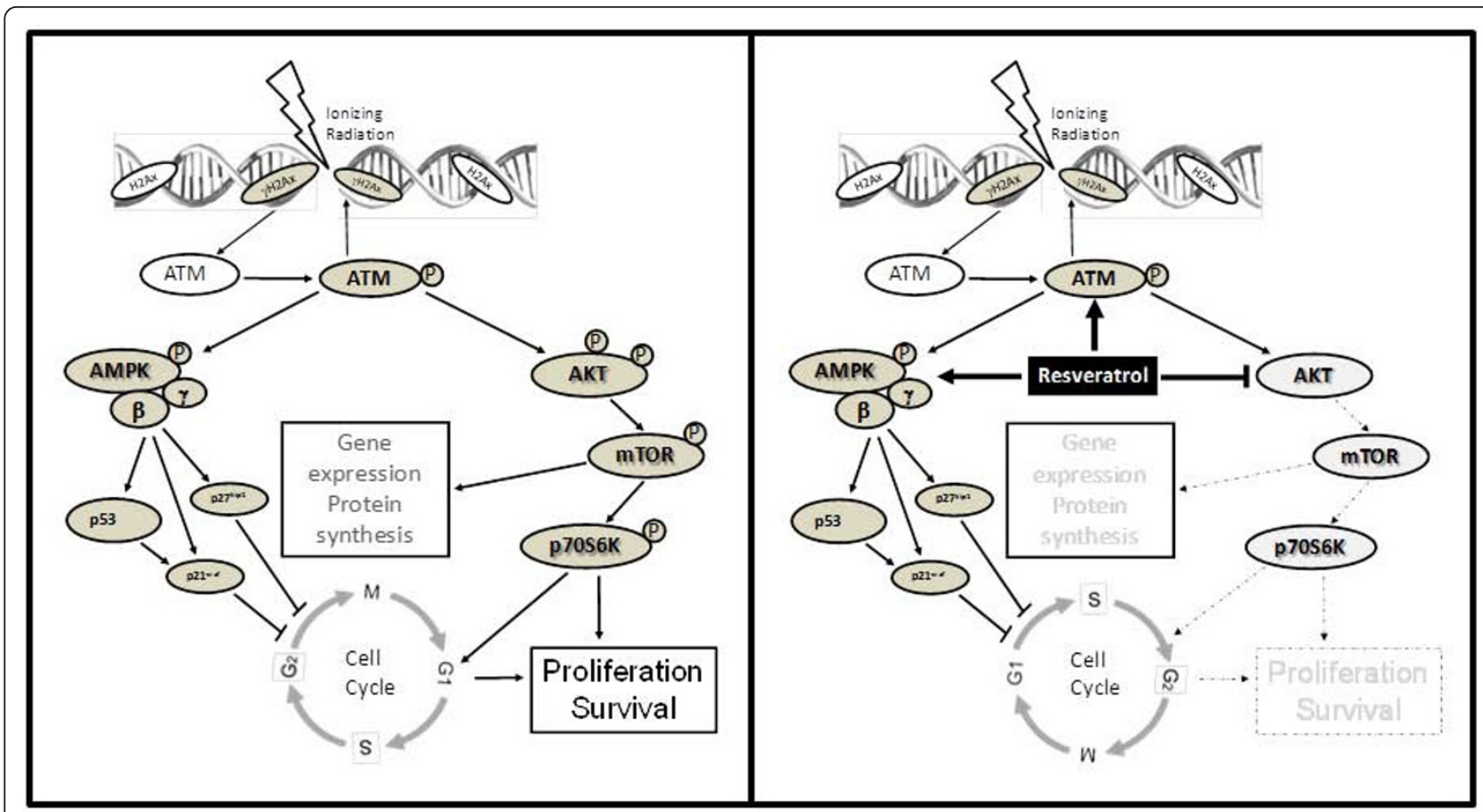

Figure 7 Model of the mechanism of action of resveratrol (RSV) and ionizing radiation (IR) to regulate cell cycle and survival in PrCa cells. A. IR induces DNA strand breaks which are detected by ATM, leading to AMPK activation and induction of the cell cycle inhibitors p53, $\mathrm{p} 21^{\mathrm{cip} 1}$ and $\mathrm{p} 27^{\mathrm{kip} 1}$. IR also activates Akt to increase protein synthesis through mTOR and stimulate cell cycle progression, proliferation and survival. B. The work presented here suggests that RSV i) enhances the effects of IR on AMPK activation, leading to an early cell cycle arrest, and ii) inhibits Akt to reduce gene expression and proliferation and enhance radiation cytotoxicity.

\section{Conclusions}

Figure 7 illustrates a model of the effects of RSV on the IR responses of PrCa cells. Low $(\mu \mathrm{M})$ concentrations of RSV, that can be achieved in human serum, inhibit PrCa cell survival and enhance IR-induced cytotoxicity. RSV enhances IR-induced DNA-damage response signals (ATM), apoptosis markers (cleaved caspase-3) and cell cycle inhibitors (p53, p21 $1^{\text {cip } 1}$ and p2 $\left.7^{\text {kip } 1}\right)$. Further, it arrests cycling at the G1-S phase and enhances IRinduced nuclear aberrations. RSV exerts a desirable dual action to inhibit the Akt radioresistance pathway while enhancing the tumour suppressor AMPK, leading to potentiation of an ATM-AMPK-p53-p21 $1^{\text {cip1 }} / \mathrm{p} 27^{\text {kip1 }}$ signaling axis. We conclude that RSV is a promising agent that deserves further investigation as an adjunct to IR in in-vivo models of PrCa to help elucidate its potential for clinical use in combination with RT.

\section{Acknowledgements}

This work was supported by funds from the Canadian Association of Radiation Oncologists (CARO - Abbott Laboratories ACURA Award) and the Canadian Institutes of Health Research (ClHR).

\section{Author details}

${ }^{1}$ Translational Radiation Biology Laboratory, Juravinski Cancer Centre, 699 Concession Street, Hamilton, Ontario, L84 5C2, Canada. ${ }^{2}$ Department of Oncology, McMaster University, 1280 Main Street West, Hamilton, Ontario,
L8S 4L8, Canada. ${ }^{3}$ Department of Pathology and Molecular Medicine, McMaster University, 1280 Main Street West, Hamilton, Ontario, L8S 4L8, Canada. ${ }^{4}$ Department of Community Health Science, Brock University, 500 Glenridge Avenue, St. Catharines, Ontario, L2S 3A1, Canada. ${ }^{5}$ Department of Radiation Oncology, Princess Margaret Hospital, University of Toronto, 610 University Avenue, Toronto, Ontario, M5G 2M9, Canada.

\section{Authors' contributions}

AR carried out the cellular/molecular experiments (clonogenic assays), cell cycle studies and helped draft the manuscript. CL aided in the cell cycle studies. TS helped carry out the immunofluorescence experiments. $\pi$ conceived the study, directed the study design, supervised all experimental work and prepared the manuscript. ET, GS, RB participated in the design of the study and advised $\Pi$ on experimental approaches. All authors have read and approved the final manuscript.

\section{Competing interests}

The authors declare that they have no competing interests.

Received: 4 August 2011 Accepted: 26 October 2011

Published: 26 October 2011

\section{References}

1. Zietman AL, Bae K, Slater JD, Shipley WU, Efstathiou JA, Coen JJ, Bush DA, Lunt M, Spiegel DY, Skowronski R, Jabola BR, Rossi CJ: Randomized trial comparing conventional-dose with high-dose conformal radiation therapy in early-stage adenocarcinoma of the prostate: long-term results from proton radiation oncology group/american college of radiology 9509. J Clin Oncol 2010, 28(7):1106-1111.

2. Cantley LC: The phosphoinositide 3-kinase pathway. Science 2002, 296(5573):1655-1657.

3. Rodemann HP, Dittmann K, Toulany M: Radiation-induced EGFR-signaling and control of DNA-damage repair. Int J Radiat Biol 2007, 83(1112):781-791. 
4. Burgering BM, Coffer PJ: Protein kinase B (c-Akt) in phosphatidylinositol3-OH kinase signal transduction [see comments]. Nature 1995, 376(6541):599-602.

5. Alessi DR, James $S R$, Downes $C P$, Holmes $A B$, Gaffney $P R$, Reese $C B$, Cohen P: Characterization of a 3-phosphoinositide-dependent protein kinase which phosphorylates and activates protein kinase Balpha. Curr Biol 1997, 7(4):261-269.

6. Viniegra JG, Martinez N, Modirassari P, Losa JH, Parada Cobo C, Lobo VJ, Luquero Cl, Alvarez-Vallina L, Ramon y, Cajal S, Rojas JM, Sanchez-Prieto R: Full activation of PKB/Akt in response to insulin or ionizing radiation is mediated through ATM. J Biol Chem 2005, 280(6):4029-4036.

7. Nelson EC, Evans CP, Mack PC, Devere-White RW, Lara PN Jr: Inhibition of Akt pathways in the treatment of prostate cancer. Prostate Cancer Prostatic Dis 2007, 10(4):331-339.

8. Mayo LD, Donner DB: A phosphatidylinositol 3-kinase/Akt pathway promotes translocation of $\mathrm{Mdm} 2$ from the cytoplasm to the nucleus. Proc Natl Acad Sci USA 2001, 98(20):11598-11603.

9. Li Y, Dowbenko D, Lasky LA: AKT/PKB phosphorylation of p21Cip/WAF1 enhances protein stability of p21Cip/WAF1 and promotes cell survival. J Biol Chem 2002, 277(13):11352-11361.

10. Viglietto G, Motti ML, Bruni P, Melillo RM, D'Alessio A, Califano D, Vinci F, Chiappetta G, Tsichlis P, Bellacosa A, Fusco A, Santoro M: Cytoplasmic relocalization and inhibition of the cyclin-dependent kinase inhibitor p27(Kip1) by PKB/Akt-mediated phosphorylation in breast cancer. Nat Med 2002, 8(10):1136-1144

11. Contessa JN, Hampton J, Lammering G, Mikkelsen RB, Dent P, Valerie $K$, Schmidt-Ullrich RK: lonizing radiation activates Erb-B receptor dependent Akt and p70 S6 kinase signaling in carcinoma cells. Oncogene 2002, 21(25):4032-4041.

12. Diaz R, Nguewa PA, Diaz-Gonzalez JA, Hamel E, Gonzalez-Moreno O, Catena R, Serrano D, Redrado M, Sherris D, Calvo A: The novel Akt inhibitor Palomid 529 (P529) enhances the effect of radiotherapy in prostate cancer. Br J Cancer 2009, 100(6):932-940.

13. Steinberg GR, Kemp BE: AMPK in Health and Disease. Physiol Rev 2009, 89(3):1025-1078.

14. Jones RG, Plas DR, Kubek S, Buzzai M, Mu J, Xu Y, Birnbaum MJ, Thompson CB: AMP-activated protein kinase induces a p53-dependent metabolic checkpoint. Mol Cell 2005, 18(3):283-293.

15. Sanli T, Rashid A, Liu C, Harding S, Bristow RG, Cutz JC, Singh G, Wright J, Tsakiridis T: lonizing radiation activates AMP-activated kinase (AMPK): a target for radiosensitization of human cancer cells. Int I Radiat Oncol Biol Phys 2010, 78(1):221-229.

16. Aggarwal BB, Bhardwaj A, Aggarwal RS, Seeram NP, Shishodia S, Takada Y: Role of resveratrol in prevention and therapy of cancer: preclinical and clinical studies. Anticancer Res 2004, 24(5A):2783-2840.

17. Carbo N, Costelli P, Baccino FM, Lopez-Soriano FJ, Argiles JM: Resveratrol, a natural product present in wine, decreases tumour growth in a rat tumour model. Biochem Biophys Res Commun 1999, 254(3):739-743.

18. Scarlatti F, Sala G, Ricci C, Maioli C, Milani F, Minella M, Botturi M, Ghidoni R: Resveratrol sensitization of DU145 prostate cancer cells to ionizing radiation is associated to ceramide increase. Cancer Lett 2007, 253(1):124-130.

19. Zoberi I, Bradbury CM, Curry HA, Bisht KS, Goswami PC, Roti Roti JL, Gius D: Radiosensitizing and anti-proliferative effects of resveratrol in two human cervical tumor cell lines. Cancer Lett 2002, 175(2):165-173.

20. Um JH, Park SJ, Kang H, Yang S, Foretz M, McBurney MW, Kim MK, Viollet B, Chung JH: AMPK-deficient mice are resistant to the metabolic effects of resveratrol. Diabetes 2009.

21. Kimura Y, Okuda H: Resveratrol isolated from Polygonum cuspidatum root prevents tumor growth and metastasis to lung and tumor-induced neovascularization in Lewis lung carcinoma-bearing mice. J Nutr 2001, 131(6):1844-1849.

22. Aziz MH, Nihal M, Fu VX, Jarrard DF, Ahmad N: Resveratrol-caused apoptosis of human prostate carcinoma LNCaP cells is mediated via modulation of phosphatidylinositol 3'-kinase/Akt pathway and BCl-2 family proteins. Mol Cancer Ther 2006, 5(5):1335-1341.

23. Zang M, Xu S, Maitland-Toolan KA, Zuccollo A, Hou X, Jiang B, Wierzbicki M, Verbeuren TJ, Cohen RA: Polyphenols stimulate AMP-activated protein kinase, lower lipids, and inhibit accelerated atherosclerosis in diabetic LDL receptor-deficient mice. Diabetes 2006, 55(8):2180-2191.
24. Hwang J-T, et al: Resveratrol induces apoptosis in chemoresistant cancer cells via modulation of AMPK signaling pathways. Ann NY Acad Sci 2007, 1095(1):441-448.

25. Sexton E, Van Themsche C, LeBlanc K, Parent S, Lemoine P, Asselin E: Resveratrol interferes with AKT activity and triggers apoptosis in human uterine cancer cells. Mol Cancer 2006, 5:45.

26. Sabolic I, Brown D: Water channels in renal and nonrenal tissues. NIPS 1995, 10:12-17.

27. Bromfield GP, Meng A, Warde P, Bristow RG: Cell death in irradiated prostate epithelial cells: role of apoptotic and clonogenic cell kill. Prostate Cancer Prostatic Dis 2003, 6(1):73-85.

28. Sramkoski RM, Pretlow TG, Giaconia JM, Pretlow TP, Schwartz S, Sy MS, Marengo SR, Rhim JS, Zhang D, Jacobberger JW: A new human prostate carcinoma cell line, 22Rv1. In Vitro Cell Dev Biol Anim 1999, 35(7):403-409.

29. Bussink J, van der Kogel AJ, Kaanders JH: Activation of the PI3-K/AKT pathway and implications for radioresistance mechanisms in head and neck cancer. Lancet Oncol 2008, 9(3):288-296.

30. Hickson I, Zhao Y, Richardson CJ, Green SJ, Martin NM, Orr Al, Reaper PM, Jackson SP, Curtin NJ, Smith GC: Identification and characterization of a novel and specific inhibitor of the ataxia-telangiectasia mutated kinase ATM. Cancer Res 2004, 64(24):9152-9159.

31. Gescher AJ, Steward WP: Relationship between mechanisms, bioavailibility, and preclinical chemopreventive efficacy of resveratrol: a conundrum. Cancer Epidemiol Biomarkers Prev 2003, 12(10):953-957.

32. Shankar S, Singh G, Srivastava RK: Chemoprevention by resveratrol: molecular mechanisms and therapeutic potential. Front Biosci 2007, 12:4839-4854.

33. Skvortsova I, Skvortsov S, Stasyk T, Raju U, Popper BA, Schiestl B, von Guggenberg E, Neher A, Bonn GK, Huber LA, Lukas P: Intracellular signaling pathways regulating radioresistance of human prostate carcinoma cells. Proteomics 2008, 8(21):4521-4533.

34. Li L, Ross AH: Why is PTEN an important tumor suppressor? J Cell Biochem 2007, 102(6):1368-1374.

35. Graff JR, Konicek BW, McNulty AM, Wang Z, Houck K, Allen S, Paul JD, Hbaiu A, Goode RG, Sandusky GE, Vessella RL, Neubauer BL: Increased AKT activity contributes to prostate cancer progression by dramatically accelerating prostate tumor growth and diminishing p27Kip1 expression. J Biol Chem 2000, 275(32):24500-24505.

36. Hsieh TC, Wu JM: Differential effects on growth, cell cycle arrest, and induction of apoptosis by resveratrol in human prostate cancer cell lines. Exp Cell Res 1999, 249(1):109-115.

37. Rusin M, Zajkowicz A, Butkiewicz D: Resveratrol induces senescence-like growth inhibition of U-2 OS cells associated with the instability of telomeric DNA and upregulation of BRCA1. Mech Ageing Dev 2009, 130(8):528-537.

38. Ahmad N, Adhami VM, Afaq F, Feyes DK, Mukhtar H: Resveratrol causes WAF-1/p21-mediated G(1)-phase arrest of cell cycle and induction of apoptosis in human epidermoid carcinoma A431 cells. Clin Cancer Res 2001, 7(5):1466-1473.

39. Gill C, Walsh SE, Morrissey C, Fitzpatrick JM, Watson RW: Resveratrol sensitizes androgen independent prostate cancer cells to death-receptor mediated apoptosis through multiple mechanisms. Prostate 2007, 67(15):1641-1653.

40. Shih A, Zhang S, Cao HJ, Boswell S, Wu YH, Tang HY, Lennartz MR, Davis FB, Davis PJ, Lin HY: Inhibitory effect of epidermal growth factor on resveratrol-induced apoptosis in prostate cancer cells is mediated by protein kinase C-alpha. Mol Cancer Ther 2004, 3(11):1355-1364.

41. Castedo M, Perfettini JL, Roumier T, Andreau K, Medema R, Kroemer G: Cell death by mitotic catastrophe: a molecular definition. Oncogene 2004, 23(16):2825-2837.

42. Gurumurthy S, Vasudevan KM, Rangnekar VM: Regulation of apoptosis in prostate cancer. Cancer Metastasis Rev 2001, 20(3-4):225-243.

43. Liang J, Shao SH, Xu ZX, Hennessy B, Ding Z, Larrea M, Kondo S, Dumont DJ, Gutterman JU, Walker CL, Slingerland JM, Mills GB: The energy sensing LKB1-AMPK pathway regulates p27(kip1) phosphorylation mediating the decision to enter autophagy or apoptosis. Nat Cell Biol 2007, 9(2):218-224.

doi:10.1186/1748-717X-6-144

Cite this article as: Rashid et al:: Resveratrol enhances prostate cancer cell response to ionizing radiation. Modulation of the AMPK, Akt and mTOR pathways. Radiation Oncology 2011 6:144. 